\title{
In vivo auditory nerve stimulation with visible light
}

\author{
Tian Guan* \\ Graduate School at Shenzhen, Tsinghua University \\ Shenzhen 518055, P. R. China \\ guantian@sz.tsinghua.edu.cn \\ Muqun Yang \\ Tsinghua-Berkeley Shenzhen Institute, Tsinghua University \\ Shenzhen 518055, P. R. China \\ ymq14@mails.tsinghua.edu.cn \\ Yonghong $\mathrm{He}$ \\ Graduate School at Shenzhen, Tsinghua University \\ Shenzhen 518055, P. R. China \\ heyh@sz.tsinghua.edu.cn
}

Received 15 June 2017

Accepted 10 August 2017

Published 23 August 2017

\begin{abstract}
Background: Infrared laser stimulation has been proposed as an innovative method to elicit an auditory nerve response. Most studies have focused on using long-wavelength infrared (>980 nm) pulsed lasers with high water absorption coefficients. This paper sought to assess whether a shortwavelength laser $(465 \mathrm{~nm})$ with an absorption coefficient as low as $10^{-3} \mathrm{~cm}^{-1}$ would activate the auditory nerve and studied its potential mechanism. Method: Optical compound action potentials (OCAPs) were recorded when synchronous trigger laser pulses stimulate the cochlea before and after deafening, varying the pulse durations (from $800 \mu$ s to $3600 \mu \mathrm{s}$ ) and the amount of radiant energy (from $18.05 \mathrm{~mJ} / \mathrm{cm}^{2}$ to $107.91 \mathrm{~mJ} / \mathrm{cm}^{2}$ ). A thermal infrared imager was applied to monitor the temperature change of the guinea pig cochlea. Results: The results showed that pulsed laser stimulation at $465 \mathrm{~nm}$ could invoke OCAPs and had a similar waveform compared to the acoustical compound action potentials. The amplitude of OCAPs had a positive correlation with the increasing laser peak power, while the latency of OCAPs showed a negative correlation. The imager data showed that the temperature in the cochlea rose quickly by about $0.3^{\circ} \mathrm{C}$ right after stimulating the cochlea and decreased quickly back to the initial temperature as the stimulation ended. Conclusions: This paper demonstrates that 465-nm laser stimulation can successfully induce OCAPs outside the cochlea, and that the amplitude and latency of the invoked OCAPs are highly affected
\end{abstract}

${ }^{*}$ Corresponding author.

This is an Open Access article published by World Scientific Publishing Company. It is distributed under the terms of the Creative Commons Attribution 4.0 (CC-BY) License. Further distribution of this work is permitted, provided the original work is properly cited. 
by laser peak power. This paper proposes that a photothermal effect might be the main mechanism for the auditory nerve response induced by short-wavelength laser stimulation.

Keywords: Pulsed laser; optical stimulation; cochlear implant; optical compound action potentials.

\section{Introduction}

According to the World Health Organization, 360 million people suffer from disabling hearing impairment, which can cause social isolation, reduced professional capabilities, and other social problems. ${ }^{1}$ The National Institutes of Health has estimated that electrical stimulation-based cochlear implants have restored hearing for $\sim 324,200$ patients. $^{2}$ However, electrical stimulation has many disadvantages, such as low spatial resolution and intrinsic artifacts. $^{3-5}$ Optical stimulation offers a fundamental advantage over electrical stimulation in hearing prostheses by dramatically improving the frequency resolution in sound coding. ${ }^{1,6}$

Pulsed infrared neural stimulation (INS) and optogenetic approaches have been investigated as alternative methods to electrical stimulation to invoke the auditory nerve response. ${ }^{7-10}$ Arvanitki et al. were the first to determine how nerve cells were affected by different wavelengths of radiation, from visible to near-infrared light. ${ }^{11}$ Wells and colleagues obtained nerve and muscle potentials during pulsed low-energy infrared laser stimulation in a rat sciatic nerve model. They suggested that direct neural activation was induced by a transient thermal signal. ${ }^{12,13}$ Izzo et al. elicited optical compound action potentials (OCAPs) in the auditory nerves of deafened animals, using a Ho:YAG laser with a wavelength of $2.12 \mu \mathrm{m}$ and pulse duration of $250 \mu \mathrm{s}$. They also examined the safety of optical stimulation and parameters including pulse duration, repetition rate and wavelength in gerbil cochlea in vivo. ${ }^{6,14,15}$ Applying electrical stimulation and INS to the cochlear nucleus in a rat model, Verma et al. found that only electrical stimulation could invoke auditory brainstem responses after deafening. They suggested that the mechanism of INS was due to a laser-induced acoustic artifact. ${ }^{16}$ Friedberger and Ren were the first to apply $813 \mathrm{~nm}$ pulsed laser and researched on the vibration of basilar membrane. They demonstrated that a moderately powerful laser could deliver enough energy to stimulate the hearing organ. ${ }^{17}$ Tan et al. adopted customdesigned angle polished fibers to deliver photons and stimulate the cochlea. Mean radiant exposure required to evoke OCAPs was $18.9-12.2 \mathrm{~mJ} /$ $\mathrm{cm}^{2} .{ }^{18,19}$ The authors proposed that their results supported the notion of direct radiant interaction with neurons. Kallweit et al. measured the pressure evoked by pulsed laser stimulating a water cylinder and the corresponding signal shapes of laser absorption in intracochlear optical stimulation of guinea pigs. They proposed an optoacoustic effect as the basic mechanism of INS. ${ }^{20}$

The cochlea is a conical, lymph-filled chamber. Photons from pulsed lasers must pass through lymph and other tissues before reaching the auditory nerve. Long-wavelength lasers have relatively high water absorption coefficients. These lasers suffer energy loss during cochlear stimulation due to energy absorption by lymph or other tissues, which can cause heat accumulation and might invoke photothermal and optoacoustic effects. For better induction of the auditory nerve, the laser output has to be increased, which might cause thermal damage to the tissues. Short-wavelength lasers have lower water absorption coefficients. ${ }^{21}$ Thus, the use of short-wavelength lasers could reduce the energy loss due to lymph absorption and enable more energy to reach the auditory nerve.

In this paper, we used a pulsed laser with a wavelength of $465 \mathrm{~nm}$ to stimulate the cochlea of guinea pigs. We analyzed the optical and acoustic compound action potentials (ACAPs) to determine whether stimulation at $465 \mathrm{~nm}$ could invoke an auditory response in deafened cochlea, and monitored the temperature change during stimulation to analyze the mechanism of generating OCAPs.

\section{Materials and Methods}

\subsection{Schematic diagram}

Figure 1 is the schematic diagram of the optical experiment. Output power of the laser (LSR465CPFC-3W) was modulated by a current source controller (LSR-PS-FA, Ningbo Yuan Ming Laser Technology Co. Ltd., Ningbo, China). Pulse duration was regulated by a microcontroller unit (MC9S12XS128, Freescale Semiconductor, Inc.). Output power measured at the distal end of the 


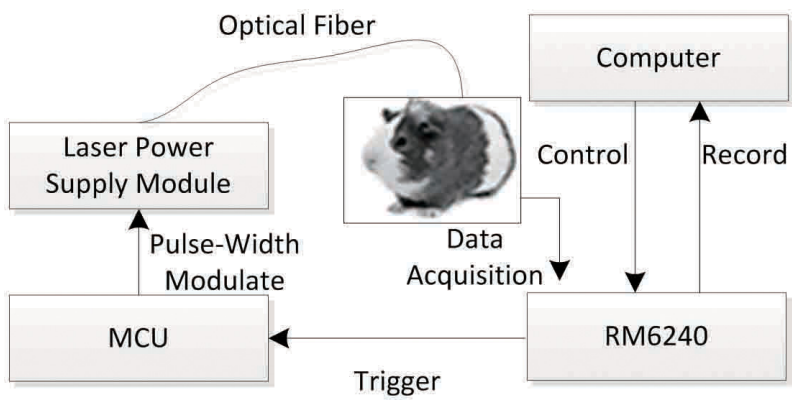

Fig. 1. Schematic diagram of the optical experiment. The pulse width was modulated by microcontroller unit (MCU). The output power was measured at the distal end of the fiber, ranging from $0 \mathrm{~W}$ to $2.3 \mathrm{~W}$. A physiological signal acquisition system (RM6240C, Chengdu Instrument Factory, and Chengdu, China) was used to collect the signals and then transmitted to the computer.

fiber ranged from $0 \mathrm{~W}$ to $2.3 \mathrm{~W}$. A physiological signal acquisition system (RM6240C, Chengdu Instrument Factory, Chengdu, China) was used to trigger the laser source synchronously and acquire signals. A thermal infrared imager (Thermo Vision SC6000, FLIR Systems Inc., USA) was used to measure temperature changes of the cochlea during the experiment.

\subsection{Stimulation and signal acquisition}

Acoustical stimuli are generated by a speaker (SPA2380/93, Philips Investment Co. Ltd., Shanghai, China) to present a tone burst at a repetition rate of $0.5 \mathrm{~Hz}$ and pressure of $82 \mathrm{~dB}$ sound pressure level (SPL). Optical stimuli are generated by a 465-nm diode laser, with radiant exposures ranging from $31.51 \mathrm{~mJ} / \mathrm{cm}^{2}$ to $107.91 \mathrm{~mJ} / \mathrm{cm}^{2}$ and pulse durations ranging from $800 \mu \mathrm{s}$ to $3.6 \mathrm{~ms}$. ACAPs and OCAPs are measured by a physiological signal acquisition system at a sampling rate of $100 \mathrm{kHz}$. For each animal, 20 measurements of CAP signals are averaged to reduce the influence of random error, obtaining a final CAP signal that is analyzed offline in MATLAB. Temperature data is analyzed in Flir R\&D Software 3.3 (FLIR Systems Inc.) and MATLAB.

\subsection{Anesthesia and surgery}

All procedures were conducted in accordance with the guidelines of the Administrative Committee on Animal Research at the Graduate School at Shenzhen, Tsinghua University. Ten adult guinea pigs of either sex (weight: 200-300 g) were divided into group 1 or group 2. Group 1 would be acutely deafened by streptomycin sulfate solution while group 2 have been chronically deafened by injecting streptomycin sulfate solution for 1 month. All experiments were conducted in an anechoic chamber.

Animals were anesthetized by an initial intraperitoneal injection of $20 \%$ ethyl carbamate $(6 \mathrm{ml} / \mathrm{kg}$ body weight) before the experiment, followed by maintenance doses (about $3 \mathrm{ml} / \mathrm{kg}$ body weight) given every hour as required after paw pinch assessment. The animal was positioned on a heating pad (BORO, BR Pet Products Co. Ltd., Dongguan, China) to maintain the body temperature at $38^{\circ} \mathrm{C}$. The mastoid bone beside the skull was exposed by cutting off the pinna. A hole $(\sim 2-\mathrm{mm}$ diameter) was drilled in the mastoid bone to allow the access to the cochlea. The hole was enlarged until a round window was visible. A silver recording electrode (XF100, Chengdu Instrument Factory) was placed at the promontorium tympani near the round window. A reference electrode was clamped to the auricular skin, and a ground electrode was inserted into the dorsal skin of the animal.

After measuring the ACAPs, extensive surgery was done to deafen all animals by removing the tympanic membrane and ossicular chain. For group 2 , animals were additionally injected with $10 \mu \mathrm{L}$ of

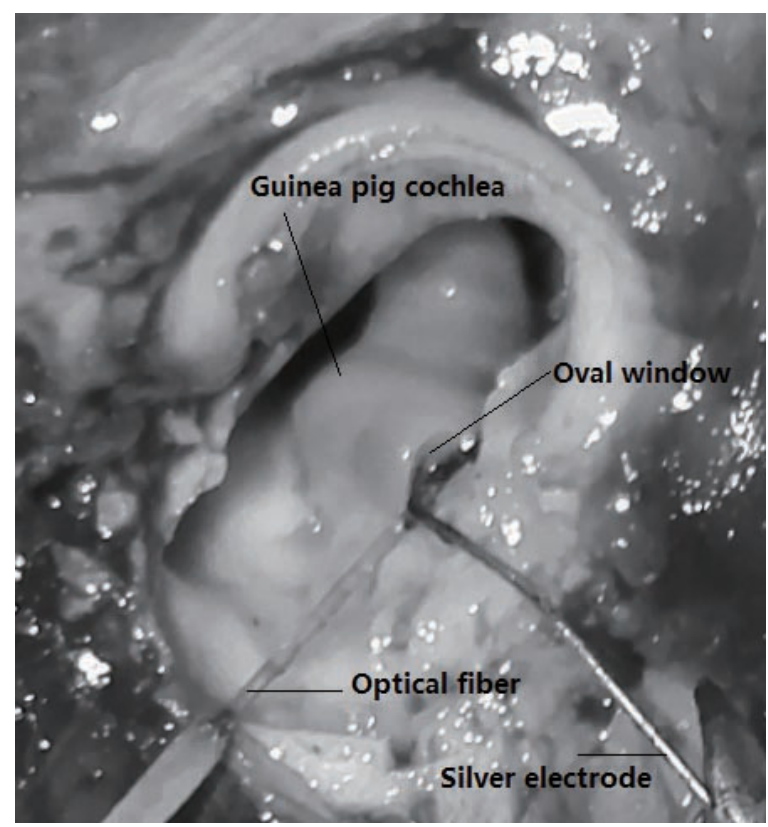

Fig. 2. Relative location among the optical fiber, silver electrode and cochlea. 
$2.5 \%$ streptomycin sulfate solution (Dalian Merro Pharmaceutical Factory, Batch no. 2001 0905, China). Then, optical experiments were conducted. Laser output was coupled to an optical fiber (200- $\mu \mathrm{m}$ diameter), which was fixed on a micromanipulator (MP-225, Sutter Instrument Co., Beijing, China). The end of the optical fiber was placed near the round window membrane, visually oriented toward the spiral ganglion cells in Rosenthal canal in the basal turn without making a hole in the cochlea. Relative locations of the optical fiber, silver electrode and cochlea are shown in Fig. 2. We recorded signals while performing direct radiant stimulation onto the recording electrode to study whether radiance applied to an electrode evokes CAPs.

\section{Results}

\subsection{Comparison between $A C A P s$ and $\mathrm{OCAPs}$}

ACAPs for a guinea pig before and after deafening surgery are shown in Fig. 3. ACAP amplitude between $\mathrm{N} 1$ and $\mathrm{P} 1$ was $230 \mu \mathrm{V}$ before deafening. Very small signals of ACAPs were invoked after deafening surgery which verified the effectiveness of the deafening surgery. Signals for direct optical stimulation to the recording electrode were relatively smooth, and no CAPs were observed.

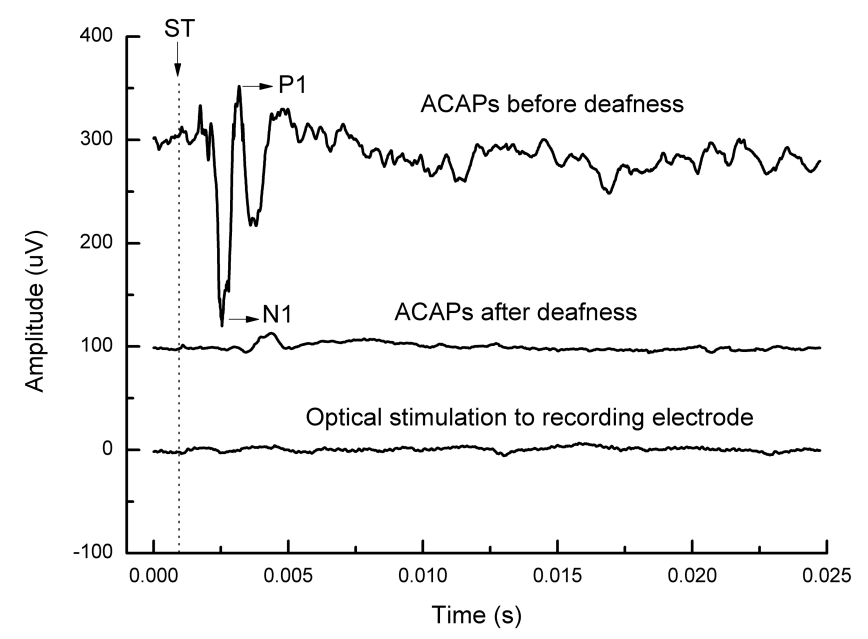

Fig. 3. ACAPs from the guinea pig before and after deafening surgery were measured with a tone burst by a speaker at $82 \mathrm{~dB}$ SPL and operated at a repetition rate of $0.5 \mathrm{~Hz}$. The curve at the bottom is the signal through optical stimulation directly to the record electrode with black cloth covering the cochlear. ST stands for the starting time of stimulation.

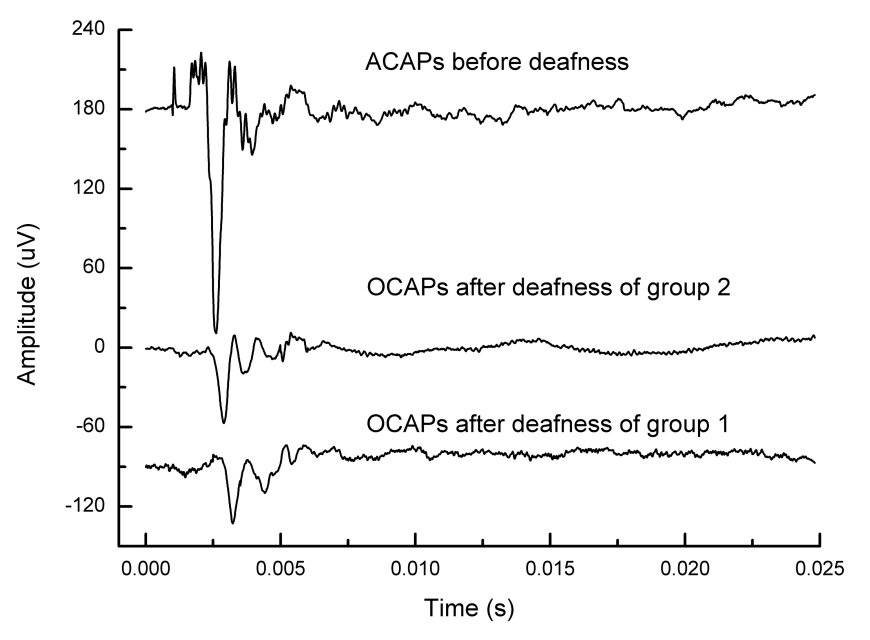

Fig. 4. The upper curve shows ACAPs measured in response to acoustic stimulation before deafness with acoustic tone bursts at $82 \mathrm{~dB}$ SPL; The lower two curves show OCAPs measured in response to $465 \mathrm{~nm}$ laser stimulation after deafening in both groups.

Figure 4 shows ACAPs measured before and OCAPs measured after deafness was induced in a guinea pig. ACAP and OCAP curves had latencies ranging from $\sim 1.0 \mathrm{~ms}$ to $2.8 \mathrm{~ms}$. ACAP amplitude was $\sim 230 \mu \mathrm{V}$ prior to deafness, while very small signals of ACAP were recorded after deafness. OCAPs with amplitudes ranging from $45 \mu \mathrm{V}$ to $60 \mu \mathrm{V}$ were successfully induced after deafness. Cochlear microphonic potentials were observed in ACAP curves before deafness, whereas no cochlear microphonic potentials were observed in OCAP curves in either group.

\subsection{OCAPs with different radiant exposures}

We measured OCAPs while gradually increasing radiant exposure at the distal end of the optical fiber from $31.51 \mathrm{~mJ} / \mathrm{cm}^{2}$ to $107.91 \mathrm{~mJ} / \mathrm{cm}^{2}$ (Fig. 5). In general, OCAP amplitudes increased rapidly with increasing radiant exposure when energy density was below $58.25 \mathrm{~mJ} / \mathrm{cm}^{2}$, but plateaued when radiant exposure was further increased. OCAP latencies ( $N 1$ times) decreased from $2.2 \mathrm{~ms}$ to $1.9 \mathrm{~ms}$ when radiant exposure was increased. On average, when compared to group 1, amplitudes in group 2 were lower, but latencies were $\sim 0.05 \mathrm{~ms}$ higher. One-way ANONA result showed that amplitudes of OCAPs between groups 1 and 2 were not significantly different at the 0.05 level. $(P=0.519)$. 


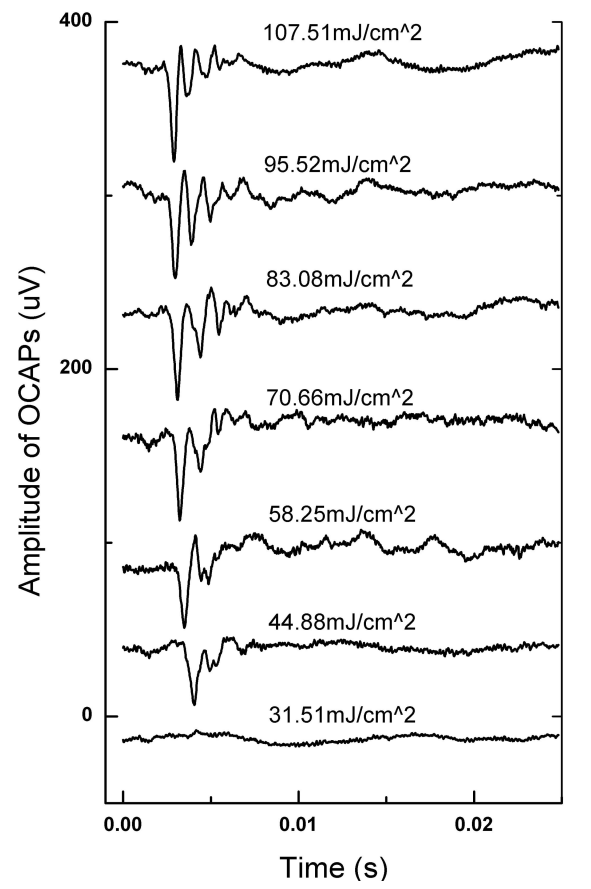

(a)

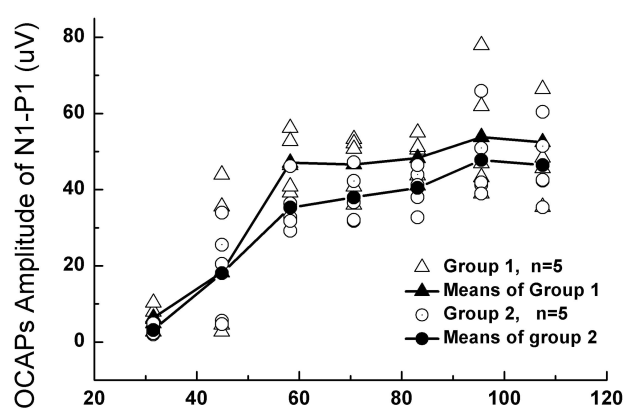

(b)

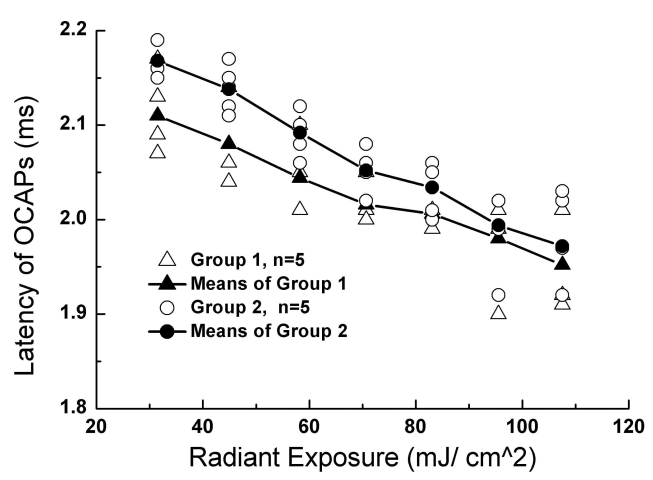

(c)

Fig. 5. (a) Optical stimulated CAPs obtained upon increasing the radiant exposure at the end of the optical fiber from $31.51 \mathrm{~mJ} / \mathrm{cm}^{2}$ to $107.51 \mathrm{~mJ} / \mathrm{cm}^{2}$. (b, c) The amplitudes of $N 1-P 1$ and latency of $N 1$ for all animals in two groups are plotted as hollow triangles and hollow circles, respectively. Solid triangles and circles stand for their respective means.

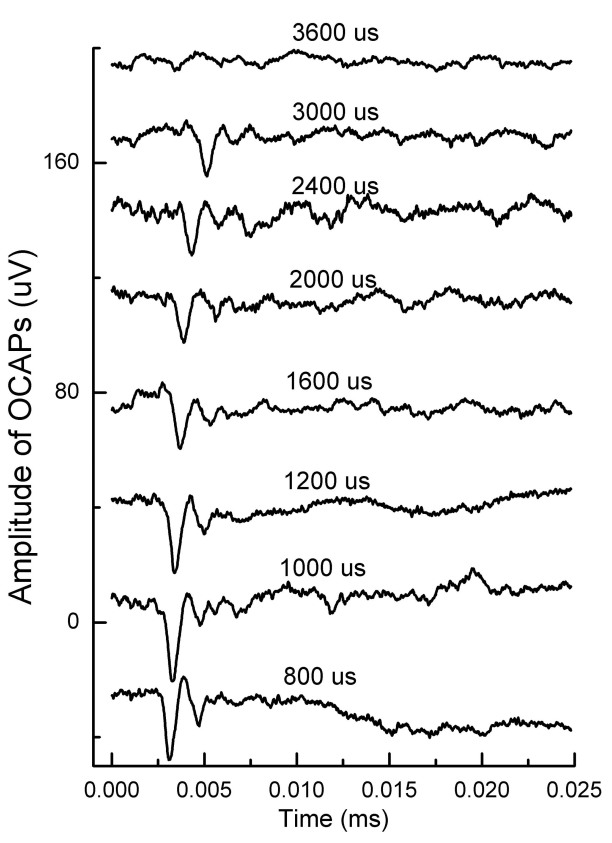

(a)

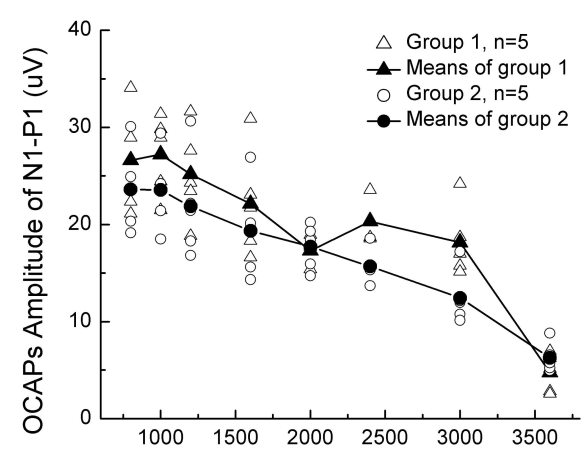

(b)

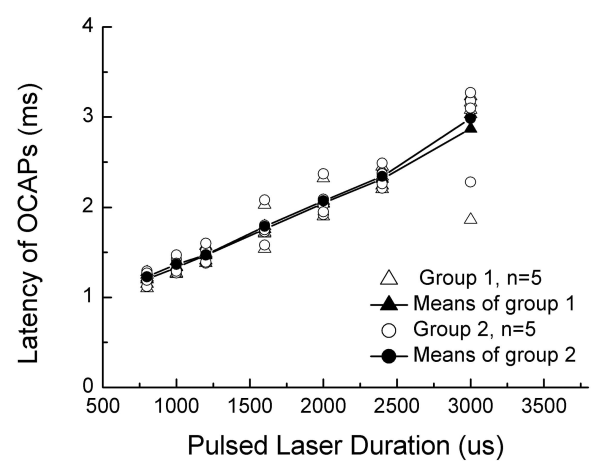

(c)

Fig. 6. (a) Optical stimulated CAPs obtained at constant radiant exposure $\left(44.88 \mathrm{~mJ} / \mathrm{cm}^{2}\right)$ with different pulse durations, from the bottom to the top: $800,1000,1200,1600,2000,2400$, 3000 and $3600 \mu \mathrm{s}$. (b, c) The amplitudes of $N 1-P 1$ and latency of $N 1$ for all animals in two groups are plotted as hollow triangles and hollow circles, respectively. Solid triangles and circles stand for their respective means. 


\subsection{OCAPs with different pulse durations}

Figure 6 depicts the effects of different pulse durations $(800-3000 \mu \mathrm{s})$ on OCAPs at constant radiant exposure $\left(44.88 \mathrm{~mJ} / \mathrm{cm}^{2}\right)$. As pulse duration increased, OCAP amplitudes decreased gradually to reach a stable value, before finally falling to zero. OCAP latencies increased continuously with increasing pulse duration. Compared to group 2, group 1 had higher amplitudes but slightly smaller latencies. One-way ANONA result showed that amplitudes of OCAPs between groups 1 and 2 were not significantly different at the 0.05 level. $(P=0.441)$.

\subsection{Temperature change of guinea pig cochlea}

Due to the limitation of the acquisition rate of the Thermo Vision SC6000 and to ensure accuracy,

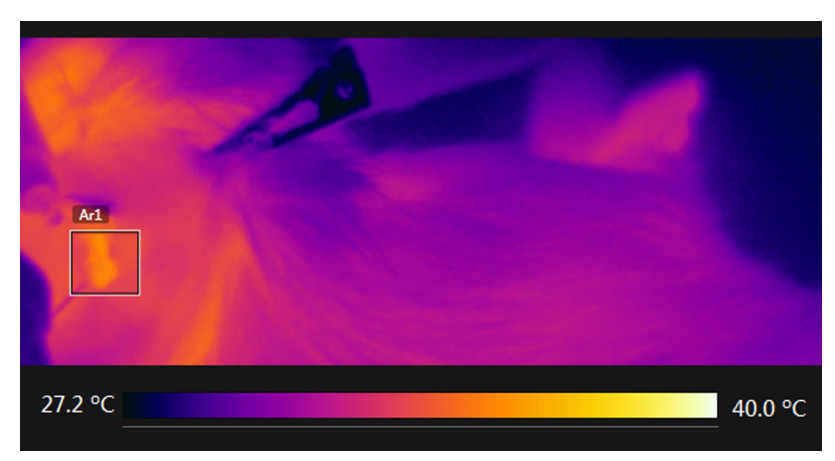

(a)

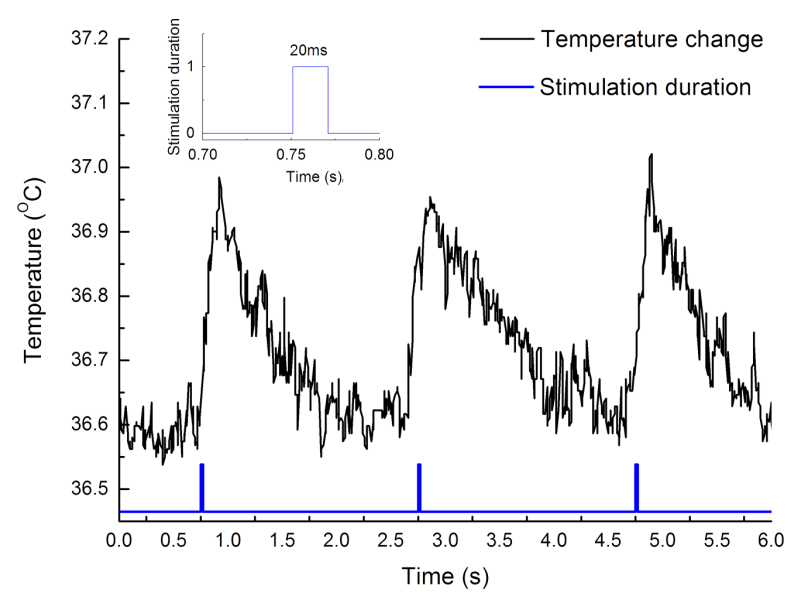

(b)

Fig. 7. (a) Depicts temperature measurement of cochlea of a guinea pig. (b) The dotted line shows the starting time of stimulation and duration is $20 \mathrm{~ms}$. The solid line shows temperature changes in the block diagram Ar1. we could only use a duration of $20 \mathrm{~ms}$ in this experiment. Figure 7 (a) depicts the temperature distribution in the guinea pig cochlea. Figure $7(\mathrm{~b})$ shows the temperature change in the block diagram of Fig. 7 (a) with a radiant exposure of $95.52 \mathrm{~mJ} /$ $\mathrm{cm}^{2}$. Cochlear temperature increased quickly by $\sim 0.35^{\circ} \mathrm{C}$ after laser stimulation, and then slowly decreased back to the initial temperature.

\section{Discussion}

The majority of previous studies on optical neural stimulation focused on long-wavelength near-infrared light. ${ }^{5-7,12-15}$ In this study, we applied a shortwavelength $(465 \mathrm{~nm})$ pulsed laser with a low water absorption coefficient to the auditory nerve in vivo. Our experiments proved that a $465-\mathrm{nm}$ pulsed laser could induce OCAPs from acutely and chronically deafened guinea pigs.

When the pulse duration was fixed at $3000 \mu \mathrm{s}$, we found that the threshold of radiant exposure to invoke OCAPs was $\sim 44.88 \mathrm{~mJ} / \mathrm{cm}^{2}$, corresponding to a pulse energy of $14.10 \mu \mathrm{J}$, and the average amplitude was $18 \mu \mathrm{V}$. Previous researchers recorded OCAP amplitudes of $12 \mu \mathrm{V}$ with radiant exposure of $\sim 1.015 \mathrm{~J} / \mathrm{cm}^{2}$ using a $808-\mathrm{nm}$ pulsed laser, ${ }^{22}$ $\sim 100 \mu \mathrm{V}$ with radiant exposure of $\sim 50 \mathrm{~mJ} / \mathrm{cm}^{2}$ using a $980-\mathrm{nm}$ laser, ${ }^{23}$ and $>300 \mu \mathrm{V}$ with radiant exposure of $100 \mathrm{~mJ} / \mathrm{cm}^{2}$ using a $1940-\mathrm{nm}$ laser. ${ }^{24}$ These aforementioned results were similar to those obtained by Schultz et al., who studied different wavelengths of laser stimulation to the inner ear. ${ }^{21}$ It might be ascribed to the increasing coefficients of water or hemoglobin absorption. ${ }^{21,25}$

With a constant radiant exposure, OCAP amplitudes decreased gradually with increasing pulse duration. The N1 latency was correlated negatively with peak power when pulse duration was constant, but correlated positively with pulse duration when radiant exposure was constant. Comparing groups 1 and 2, we observed that deafness with streptomycin sulfate solution resulted in a $\sim 10 \mu \mathrm{V}$ reduction in amplitude and a $0.05-\mathrm{ms}$ increase in latency.

Compared to the previous studies, increasing the peak power improved the amplitude of OCAP induced by $465-\mathrm{nm}$ laser light and shortened the latency. ${ }^{22-24}$ The OCAP latency induced by $465-\mathrm{nm}$ light was $\sim 2.0 \mathrm{~ms}$, compared to $>1.7 \mathrm{~ms}$ for OCAPs induced by $980-\mathrm{nm}$ light. ${ }^{21}$ Latency is defined as the time necessary for information to 
disseminate in a cytodendritic structure consisting of a hair cell and afferent nerve endings. Sound information undergoes three states (electric signalchemical signal-electric signal) in the cytodendritic structure to pass information to the auditory center and cause hearing, which takes between $1.0 \mathrm{~ms}$ and $2.0 \mathrm{~ms}$.

Animals in group 1 were acutely deafened while those in group 2 were chronically deafened. Both of these two groups were injected with the streptomycin sulfate solution. This kind of solution would damage hair cells in the cochlea. Different deafness method would lead to different damage extent. Both Figs. 4 and 5 showed no significant difference between these two groups. On the one hand, it indicates that OCAPs are not closely relative to hair cells. On the other hand, it confirms that OCAPs are more likely due to direct neural activation of pulsed laser. ${ }^{11-13}$

Despite various studies, the mechanism underlying optical stimulation of the auditory nerve remains equivocal. Many researchers have considered photochemical, photothermal, and optoacoustic effects as the most probable mechanisms of INS. ${ }^{13,21}$ For the photochemical effect, atoms, molecules, or ions in the tissue absorb the energy of photons and induce chemical reactions. For the photothermal effect, tissue absorbs the laser energy, resulting in transient heat and evoking the nerve response. For the optoacoustic effect, some substance in the tissue (e.g., water) absorbs energy from the pulsed laser and causes the temperature to rise and fall, resulting in volume expansion and shrinkage. These tissue volumetric changes could radiate sound waves outwards and, thereby, invoke CAPs.

According to the formula $E=h v=h c / \lambda$, where $E$ is the photon energy, $h$ is the Planck constant, $v$ is frequency, $c$ is the speed of light constant, and $\lambda$ is wavelength, a photochemical reaction may occur when $\lambda$ is shorter than $700 \mathrm{~nm}$. When $h$ is $6.63 \times$ $10^{-34} \mathrm{~J} \cdot \mathrm{s}$ and $c$ is $3.00 \times 10^{5} \mathrm{~km} / \mathrm{s}$, the photon energy of a $465-\mathrm{nm}$ laser is $\sim 3.38 \mathrm{eV}$. This photon energy may be sufficient to excite the atomic energy level to a higher energy state and lead to a photochemical reaction. However, the substance in the cochlea absorbs photon energy to set off a chemical reaction in a very short time (at a nanosecond level) that should not have a significant effect on the latency, in contrast to our experimental result in Fig. 6(c).
Most researchers believe that the photothermal effect is the underlying mechanism. Our experimental results clearly showed that a threshold of exposure energy of $\sim 44.88 \mathrm{~mJ} / \mathrm{cm}^{2}$ was needed to invoke OCAPs. Meanwhile, long pulse duration resulted in energy scatter to the circumambient tissue, and OCAPs were either very low amplitude or non-existent. Additionally, the temperature in the cochlea quickly increased by $\sim 0.35^{\circ} \mathrm{C}$ as the laser stimulated the cochlea, and then decreased slowly back to the initial temperature. To some extent, these verify the photothermal effect. However, to explore the potential mechanism, further work still needs to be done, including determining the hair cell damage by drug-induced deafness to further study of optoacoustic effect or researching on deeper mechanisms of photothermal effect (such as ion channels, membrane capacitance changes or temperature sensitive ion channels).

\section{Conclusion}

Using auditory neural stimulation with a 465-nm laser, we successfully invoked OCAPs in deafened guinea pigs and investigated the effects of optical parameters, including pulse energy and pulse duration on the auditory response. We propose that a photothermal effect might be the primary mechanism underlying the evoked OCAPs.

\section{Acknowledgments}

This project was supported by grants from the Nature Science Foundation of China (Nos. NSFC81401539 and NSFC31271056), Shenzhen basic research layout project (JCYJ20160324163759208) and the projects in the Shenzhen Medical Engineering Laboratory For Human Auditoryequilibrium Function.

\section{References}

1. World Health Organization, "Primary ear and hearing care training resource," World Health Organization 2 (2006).

2. G. M. Clark, "The multi-channel cochlear implant: Multi-disciplinary development of electrical stimulation of the cochlea and the resulting clinical benefit," Hear. Res. 322, 4-13 (2015).

3. L. G. Duckert, J. M. Miller, "Acute morphological changes in guinea pig cochlea following electrical 
stimulation: A preliminary scanning electron microscope study," Ann. Otol. Rhinol. Laryngol. 91(1), 33-40 (1982).

4. S. Counter, "Electromagnetic stimulation of the auditory system: Effects and side-effects," Scand. Audiol. Supp. 37, 1C32 (1993).

5. A. I. Metric, J. T. Walsh, C. P. Richter, "Spatial extent of cochlear infrared neural stimulation determined by tone-on-light masking," J. Biomed. Opt. 16(11), 118002-1180028 (2011).

6. A. D. Izzo, C. P. Richter, E. D. Jansen, J. T. Walsh, "Laser stimulation of the auditory nerve," Lasers Surg. Med. 38(8), 745-753 (2006).

7. J. M. Bec, E. S. Albert, I. Marc et al., "Characteristics of laser stimulation by near infrared pulses of retinal and vestibular primary neurons," Lasers Surg. Med. 44(9), 736-745 (2012).

8. R. L. Fork, "Laser stimulation of nerve cells in aplysia," Science 171, 907-908 (1971).

9. H. Hirase, V. Nikolenko, J. H. Goldberg, F. Yuste, "Multiphoton stimulation of neurons," J. Neurobiol. 51, 237-247 (2002).

10. J. Wells, C. Kao, K. Mariappan, J. Albea, E. D. Jansen, P. Konrad, A. M. Jansen, "Optical stimulation of neural tissue in vivo," Opt. Lett. 31, 235238 (2005).

11. A. Arvanitaki, N. Chalazonitis, "Excitatiory and inhibitory processes initiated by light and infrared radiations in single identifiable nerve cells," Nervous Inhibition, Pergamon Press, New York (1961), pp. 194-231.

12. J. Wells, C. Kao, E. D. Jansen et al., "Application of infrared light for in vivo neural stimulation," J. Biomed. Opt. 10(6), 064003 (2005).

13. J. Wells, C. Kao, P. Konrad et al., "Biophysical mechanisms of transient optical stimulation of peripheral nerve," Biophys. J. 93(7), 2567-2580 (2007).

14. A. D. Izzo, E. Suh, J. Pathria et al., "Selectivity of neural stimulation in the auditory system: A comparison of optic and electric stimuli," J. Biomed. Opt. 12(2), 021008-24000 (2007).
15. A. D. Izzo, J. T. Walsh, E. D. Jansen et al., "Optical parameter variability in laser nerve stimulation: A study of pulse duration, repetition rate, and wavelength," IEEE Trans. Biomed. Eng. 54(6), 1108-1114 (2007).

16. R. U. Verma, A. A. Guex, K. E. Hancock et al., "Auditory responses to electric and infrared neural stimulation of the rat cochlear nucleus," Hear. Res. 310, 69-75 (2014).

17. A. Fridberger, T. Ren, "Local mechanical stimulation of the hearing organ by laser irradiation," Neuroreport 17(1), 33-37 (2006).

18. X. Tan, S. Rajguru, H. Young et al., "Radiant energy required for infrared neural stimulation," Sci. Rep. 13273 (2015).

19. H. K. Young, X. Tan, N. Xia et al., "Target structures for cochlear infrared neural stimulation," Neurophotonics 2(2), 025002 (2015).

20. N. Kallweit, P. Baumhoff, A. Krueger et al., "Optoacoustic effect is responsible for laser-induced cochlear responses," Sci. Rep. (2016).

21. M. Schultz, P. Baumhoff, H. Maier et al., "Nanosecond laser pulse stimulation of the inner eara wavelength study," Biomed. Opt. Express 3(12), 3332-3345 (2012).

22. N. Xia, X. Y. Wu, X. Wang et al., "Pulsed 808-nm infrared laser stimulation of the auditory nerve in guinea pig cochlea," Lasers Med. Sci. 29(1), 343-349 (2014).

23. T. Guan, K. Zhu, F. Chen et al., "Auditory nerve impulses induced by $980 \mathrm{~nm}$ laser," J. Biomed. Opt. 20(8), 088004 (2015).

24. A. D. Izzo, J. T. Walsh, H. Ralph et al., "Laser stimulation of auditory neurons: Effect of shorter pulse duration and penetration depth," Biophys. J. 94(8), 3159-3166 (2008).

25. K. Scholle, P. Fuhrberg, P. Koopmann et al., " $2 \mathrm{~m}$ laser sources and their possible applications," INTECH Open Access Publisher 10.5772/39538 (2010). 